REVISTA ANDALUZA DE ANTROPOLOGÍA.

NÚMERO 8: TURISMO DE BASE LOCAL EN LA GLOBALIZACIÓN

MARZO DE 2015

ISSN 2174-6796

[pp. 149-154]

http://dx.doi.org/10.12795/RAA.2015.i08.09

\title{
MORENO FELIU, PAZ (2014). De lo lejano a lo próximo: un viaje por la Antropología y sus encrucijadas. : Editorial Universitaria Ramón Areces, 451 pp.
}

\author{
Sílvia Bofill Poch \\ Universidad de Barcelona
}

De lo lejano a lo próximo. Un viaje por la Antropología y sus encrucijadas, de Paz Moreno, nos ofrece un recorrido fascinante y muy original por las teorías y conceptos clásicos y modernos de la Antropología. Lejos de tratarse de un manual de historia de la antropología, se trata de un estudio sobre la diversidad humana, y sobre las alternativas críticas al racismo y la intolerancia.

El libro mantiene la estructura del anterior libro publicado por la autora (Encrucijadas antropológicas, Editorial Universitaria Ramón Areces, 2010), aunque se añaden y modifican capítulos de tal manera que el resultado es un libro distinto. Su título -De lo lejano a lo próximo- describe y al mismo tiempo evoca -en la línea de Lévi-Strauss- el recorrido histórico y conceptual que sigue el libro, mismo que ha seguido el desarrollo de la teoría antropológica desde su nacimiento en la segunda mitad del XIX hasta la actualidad.

Dicho recorrido abraza, al tiempo que problematiza, la construcción del objeto antropológico, desde unos "otros primitivos", lejanos y exóticos, aparentemente desconectados del nosotros, hasta un "nosotros" más cercano, cuyos bordes -tras ser debidamente historizados- se desdibujan, a partir de los años 70, mucho menos nítidamente que aquellos "otros", con quienes, al mismo tiempo, se confunden en múltiples encrucijadas políticas, económicas y culturales. Este recorrido se aborda 
con espíritu crítico a fin de mostrar -en palabras de la autora- "cómo se configuran en distintos momentos los saberes teóricos y analíticos de la antropología social” (p. 16).

El libro se divide en cuatro partes: 1) la primera alude a la cuestión del canon antropológico; 2) la segunda aborda la construcción del primitivo como objeto antropológico, tanto en el contexto académico como colonial; 3) la tercera reflexiona sobre las etiquetas de la diversidad cultural -etnicidad, clase, nación, género y razay sus distintos contextos sociopolíticos; 4) la cuarta y última parte aborda la práctica antropológica en situaciones extremas. Cada una de las partes se cierra con una palabra clave de Raymond Williams - cultura, naturaleza, clase y violencia- y con una selección de textos breves comentados. El libro contiene además diversos ejemplos -a modo de viñetas etnográficas- extraídos de monografías clásicas que ilustran teorías y conceptos.

En la primera parte - “Aproximación crítica al canon antropológico”- la autora rastrea críticamente la emergencia e institucionalización de la disciplina antropológica -la denominada "ciencia de los primitivos" - desde su nacimiento en el XIX hasta la década de los 70, sin olvidar algunos de sus precursores: Heródoto, Ibn Batuta y Acosta. Aquí se analizan críticamente las cuatro tradiciones nacionales básicas de la antropología: la británica, la francesa, la germánica y la norteamericana, aludiendo a sus distintos nombres y genealogías intelectuales. La cuestión nacional se muestra relevante, no sólo en la medida que la antropología ha estado ligada al devenir histórico, político e intelectual de las naciones, sino en la medida que ello ha tenido una influencia central en la delimitación de su objeto de estudio, así como en las distintas corrientes teóricas desarrolladas en el periodo clásico: evolucionismo, difusionismo, particularismo histórico, funcionalismo, estructural-funcionalismo. Todo ello -como bien nos recuerda Paz Moreno- no deja de ser problemático. Desde la publicación del trabajo de I. Hallowell, The history of anthropology as an anthropological problem (1965), hasta la más reciente Historia de la antropología. Teorías, praxis y lugares de estudio, de Martínez-Veiga (2008) (véase "El problema del canon”), pasando por el trabajo de V. Stolcke, De padre, filiaciones y malas memorias ¿Qué historias para qué antropología? (1993), sabemos que la historia de la antropología se ha convertido en un campo de investigación propio y problemático.

La crisis del modelo clásico - construido sobre la comunidad homogénea, aislada e independiente, el holismo metodológico y los parámetros clásicos del trabajo de campo expuestos por Boas y Malinowski, así como sobre un cierto sentido de ahistoricidad de las comunidades estudiadas- cierra la primera parte del libro. La constatación de que la antropología no puede desentenderse de la historia -en la línea de lo que plantearon Wolf y Roseberry, entre otros- y que aquella debe atender las múltiples y complejas interconexiones históricas y culturales, introduce la cuestión de las escalas y la denominada globalización, no sin problematizar ésta última y aludir a su carácter 
profundamente ideológico. La viñeta etnográfica sobre "Salaula: el mercado de ropa de segunda mano en Zambia" vehicula de manera muy ilustrativa la crítica hacia planteamientos clásicos dicotómicos, que ordenan y jerarquizan órdenes sociales presumiblemente situadas en distintas coordenadas espacio-temporales, al tiempo que plantean la diversidad en términos de discontinuidades y fragmentaciones esencialistas. El análisis del colonialismo y del capitalismo como procesos históricos complejos emerge aquí como un primer eje transversal del libro.

En la segunda parte del libro - "Los 'primitivos' y el colonialismo"- la autora analiza y problematiza las relaciones, múltiples y complejas, entre antropología y colonialismo. Si bien, éste es un tema crucial para la antropología, se advierte cómo el debate adquiere centralidad paradójicamente durante el periodo poscolonial, a partir de la publicación de las obras de T. Asad (Anthropology and the Colonial Encounter, 1973) y E. Saïd (Orientalismo, 1978) en la década de los 70.

Lejos de tratarse de un fenómeno homogéneo y hegemónico -como bien ilustra la "heterogeneidad cultural en la primera expansión europea" (p.197), así como los distintos "modelos de dominio colonial" (p.204)- el colonialismo debe ser entendido como un proceso diverso e históricamente localizado. Su análisis -advierte la autora siguiendo los planteamientos de antropólogos como Goody, Schumaker, Shaw o Cooper- "exige no tratarlo como si representase una categoría única o uniforme” (p. 137). Así mismo, deben ser reconocidas como diversas las relaciones construidas y localizadas entre antropólogos y pueblos colonizados.

Congruente con esta premisa, el análisis de distintas experiencias del colonialismo -y de la expansión capitalista- por parte de pueblos colonizados aparecen en el libro como extremadamente complejas ("La complejidad de las interrelaciones coloniales"); espacios de conformación de subjetividades ricas, irreductibles a un modelo único y uniforme. Las viñetas etnográficas presentadas resultan sumamente ilustrativas: rumores y acusaciones entre los Bemba contra los Padres Blancos de beber la sangre de los africanos durante el periodo colonial; auge de las acusaciones de brujería en África central y del sur en el contexto postcolonial; aparición de vampiros y otros seres monstruosos durante la década de los 80 en América Latina, especialmente en Bolivia y Perú. Con énfasis distintos, todos estos fenómenos hablan -en el marco de lo que Moreno titula "El capitalismo y sus monstruos"- de la "relación que se establece entre la riqueza, el mercado o el capitalismo con un tipo singular de sucesos extraños que amenazan la integridad del cuerpo y la vida de las personas" (p. 150).

Otro aspecto tratado aquí hace referencia a la dimensión ideológica del colonialismo. Heredera del pensamiento filosófico ilustrado, la antropología se erige sobre una de sus categorías dicotómicas fundacionales: la oposición entre "primitivos" y "civilizados". El mito de la civilización occidental subyace así, no sólo a la delimitación de su objeto, 
sino a la caracterización y clasificación del mismo. En este punto, la autora alude a uno de las categorías centrales de clasificación (política) de los pueblos estudiados por la antropología: la distinción entre sociedades igualitarias y sociedades jerarquizadas o estratificadas, es decir, con instituciones formales de gobierno. En su análisis del alcance teórico que el debate ha suscitado, contrapone los modelos de evolución socio-política (en la línea de Service y Fried) -heredados en parte de la ilustración-a modelos basados en la resistencia de las sociedades igualitarias a ser gobernadas (siguiendo a Clastres, Leach y Scott).

La tercera parte del libro - "Las etiquetas de la identidad"- rastrea las distintas aproximaciones teóricas al concepto de identidad. En alusión directa a su carácter ambiguo, la autora advierte del riesgo de no distinguir entre las categorías que usa la gente para autoadscribirse y las categorías que usan los expertos. El caso de los EEUU analizado por C. Greenhouse -donde las diferencias sociales raramente se expresan en términos de clase, y donde las definiciones antropológicas de la identidad no se corresponden con las definiciones populares de raza, clase y etnicidad- resulta ilustrativo al respecto. Remarcando el carácter procesual, fluido, múltiple y cambiante de la identidad concepto que J. Ferguson substituye por el de identificación, en alusión precisamente a su carácter histórico y procesual (Global Shadows. Africa in the Neoliberal World Order, 2006)- Moreno nos descubre el potencial analítico del concepto, así como sus múltiples tensiones e interconexiones (véase cuadro p. 239).

El ejemplo de la danza kalela desarrollado en el libro nos aproxima elocuentemente -a través de lo que algunos antropólogos como Ferguson denominan "antropología de la imitación"- al carácter histórico y cambiante de ciertas adscripciones identitarias. En un sentido similar a como lo hace el movimiento Hauka (filmado por J. Rouch) o La Sape en Brazaville (estudiada por J. Friedman), por medio de la danza kalela los Bemba y otros pueblos del Cinturón del Cobre reivindican, en pleno contexto colonial, su pertenencia a una nueva identidad basada en el significado local de la categoría "civilizado". En el trasfondo aparece la reivindicación última de compartir los mismos derechos que los europeos en una sociedad extremadamente desigual.

La identidad actúa en una doble dirección: como mecanismo de inclusión y como mecanismo de exclusión. Las categorías identitarias -clase, etnia, raza, género- se analizan aquí en relación a la desigualdad, resaltando la función ideológica que en determinados contextos históricos han tenido como fundamento de la exclusión, la opresión, la explotación y el aniquilamiento (físico o cultural) de determinados grupos y colectivos. Es el caso de conflictos basados en la estratificación étnica (y sus argumentos primordialistas), de determinadas ideologías de género que usan el sexo como argumento "natural" de exclusión e inferiorización de las mujeres o de las teorías de la raza del XIX y XX, fundamento, entre otras, de políticas raciales eugenésicas. Fenómenos recientes 
de criminalización de la población inmigrante, o de racialización de la fuerza de trabajo asociada a la feminización de los procesos migratorios en Europa y EEUU, muestran la capacidad de transformación -o transmutación- de dichas retóricas de la exclusión.

La cuarta y última parte - "La práctica antropológica ante situaciones extremas"- se focaliza en la literatura antropológica especializada en el estudio de los fenómenos de etnocidio y genocidio. En primer lugar, se señala el interés tardío que el estudio de estos fenómenos ha suscitado en la antropología y en las ciencias sociales en general. Así mismo, se advierte del riesgo analítico de focalizar los estudios en la cuestión terminológica, así como del uso restrictivo del término genocidio por parte de los países de la ONU. La complejidad de los fenómenos etnocidas y genocidas -advierte la autoraescapa a definiciones estáticas, cuyo afán de construir tipologías e inventarios dejan de lado el análisis de los procesos históricos y antropológicos que subyacen a los mismos. El caso ruandés analizado en el libro muestra de manera excelente esta complejidad, al situar el origen del genocidio Tutsi en Ruanda en el proceso histórico colonial y sus múltiples y complejas reconfiguraciones políticas e ideológicas, en este caso, vinculadas a la reconstrucción Hutu de la ideología racista europea.

El combate a actitudes y asimilaciones etnocéntricas -un segundo eje transversal del libro- pasa también por reconocer que entre nosotros también se producen y legitiman prácticas etnocidas y genocidas sobre la base de categorizaciones simbólicas asociadas a la higiene (ej. puro/impuro; limpieza/contaminación). Muestra de ello son los casos analizados de "limpieza étnica" en la antigua Yugoslavia, o de las acusaciones que tuvieron lugar en El Ejido contra los enemigos -inmigrantes- de envenenar los recursos naturales. Otros dos casos se analizan extensamente en el libro: el complejo sistema de diferenciación social basado en las categorías de "razas" o "castas" -derivado de las políticas de persecución-conversión-persecución contra judíos y moriscos- elaborado en los reinos renacentistas de los Reyes Católicos y exportado -y complejizado- al continente americano; y el sistema de las categorías de limpieza-contaminación elaborado por el régimen nacional-socialista alemán en aras a proteger la sangre aria.

El libro se cierra con una interesante y sugerente aproximación a lo que Moreno llama, siguiendo a M. Goodale (Human Rights: An Anthropological Reader, 2009), la historia de desencuentros y equívocos entre el campo de la antropología y los derechos humanos para aproximarnos a la cuestión ética -tercer y último eje transversal del libro-. Aquí se apunta la centralidad del concepto boasiano de relativismo cultural, cuya ambigüedad ética y analítica ha conllevado actitudes tan paradójicas como el rechazo de la Asociación Americana de Antropología (AAA) a firmar la Declaración de Derechos Humanos de la ONU en 1947. El debate entre universalismo y particularismo que subyace a esta actitud debe ser superado -apunta la autora- en base a la historización y contextualización, tanto de las proclamaciones de los derechos universales, como del relativismo boasiano. 
En cualquier caso, el debate sobre los derechos humanos encuentra en los años 80 un punto de inflexión. El nuevo escenario neoliberal ha propiciado la apertura de nuevas problemáticas y nuevos marcos teóricos dirigidos a comprender realidades enormemente complejas y conflictivas y, en este sentido, ha enfrentado a la comunidad antropológica a su ineludible compromiso ético con la justicia y la igualdad.

Se trata de un libro brillante, escrito con enorme sensibilidad, de obligada lectura tanto para un público experto como para el público en general. 